\title{
Essentials of Functional MRI
}

\author{
P.W. Stroman \\ Boca Raton, FL: CRC Press, 2011, 295 pages, $\$ 79.95$
}

During the last 2 decades, new developments in functional MRI have made it possible to detect changes in the brain over time, as opposed to the snapshot produced by conventional MRI. The use of functional MRI for neuroscience research has expanded rapidly since functional MRI of neural function came into existence in 1992. Clinical uses of functional MRI for diagnosis and for monitoring of therapy have been proposed, but this potential remains largely untapped in clinical practice.

Since understanding of the physiology underlying neuronal activity-related MRI signal changes improved, there has been greater agreement about the correct interpretation of functional MRI results and their reliability. We can now achieve high sensitivity to neural activity and reliably characterize changes of brain function resulting from neurologic disorders or trauma. However, functional MRI is not a single method, and thus there is no standard method that is optimal for every situation.

This handy book provides the essential information to study, understand, use, and teach the practical aspects of functional MRI for those people who are most likely to extend its use into clinical practice. The author describes the theory and practical details of functional MRI methodology, including how to acquire and analyze images. A wide range of detailed examples demonstrates how functional MRI has been used, and there is also an overview of alternative functional MRI.
This book has 9 chapters. The first two provide an introduction and describe basic concepts. Chapters 3-5 describe the source and properties of the MRI signal, the fundamental building blocks of MRI methods, and how an image is created from an MRI signal. Chapters $6-8$ deal with the principles and practice of functional MRI, the design of studies, and analysis of functional MRI data. The last chapter reviews clinical applications of functional MRI. Each chapter has valuable key points, and the book is comprehensive, combining a strong theoretic background with profound practical hints for the use of functional MRI in medical research.

This book breaks down the technical challenges for physicians, researchers, and technologists who use functional MRI but who may not be experts in the necessary math and physics. Therefore, the book is useful for both a clinical audience and MRI technologists. The more than 130 full-color figures are clear and instructive. A comprehensive glossary reviews the extensive jargon used in the MRI field. The appendix has also decision trees for BOLD (blood oxygenation level-dependent) functional MRI study design, and the references are updated.

\section{E. Edmund Kim \\ M.D. Anderson Cancer Center \\ 1400 Pressler, Unit 1483 \\ Houston, TX 77030 \\ E-mail:ekim@mdanderson.org}

COPYRIGHT (C 2012 by the Society of Nuclear Medicine and Molecular Imaging, Inc.

Published online Jul. 31, 2012.

DOI: 10.2967/jnumed.112.105452

\section{Errata}

In abstract 1559 of the 2012 abstract book (A Simplified Method for Labeling of Chondroitin Sulfate with 99mTc for Clinical Application in the Study of Osteoarthritis. J Nucl Med. 2012;53[suppl 1]:343P), a name was inadvertently omitted from the author list. The authors should read as follows: "G. Sobal, H. Ibeschitz, J. Menzel, H. Sinzinger, R. Dudczak.” We regret the error.

In the article "MRI-Based Attenuation Correction for Hybrid PET/MRI Systems: A 4-Class Tissue Segmentation Technique Using a Combined Ultrashort-Echo-Time/Dixon MRI Sequence," by Berker et al. (J Nucl Med. 2012;53:796-804), there was a transcription error in the equation calculating the water-fat fraction. The correct equation is $r=\left(M_{w}-M_{f}\right) /\left(M_{w}+M_{f}\right)$. The results of this study are not affected by this correction. We regret the error. 